Richness and relative abundance of medium and large mammals in a community of the Biosphere Reserve Tehuacán-Cuicatlán, Oaxaca, Mexico

\title{
Riqueza y abundancia relativa de mamíferos medianos y grandes en una localidad en la Reserva de la Biosfera Tehuacán-Cuicatlán, Oaxaca, México
}

\author{
Oscar Cruz-Jácome ${ }^{1}$, Eva López-Tello², Christian A. Delfín-Alfonso ${ }^{3 *}$ y Salvador Mandujano ${ }^{4}$
}

\footnotetext{
${ }^{1}$ Facultad de Biología, Universidad Veracruzana. Circuito Gonzalo Aguirre Beltrán s/n Zona Universitaria, Xalapa 91090, Veracruz, México. E- mail: borre.72@hotmail.com (OCJ)

2 Posgrado, Instituto de Neuroetología, Universidad Veracruzana. Av. Luis Castelazo Ayala s/n Col. Industrial Ánimas, Xalapa 91190, Veracruz, México. E-mail: eera_4@yahoo.com.mx (ELT)

${ }^{3}$ Instituto de Investigaciones Biológicas, Universidad Veracruzana. Av. Luis Castelazo Ayala s/n, Col. Industrial Ánimas, Xalapa 91190 Veracruz, México. E-mail: cada7305@gmail.com (CADA)

${ }^{4}$ Red de Biología y Conservación de Vertebrados, Instituto de Ecología A. C., Carretera antigua a Coatepec 351, El Haya, Xalapa 91070, Veracruz, México. E-mail: salvador.mandujano@inecol.mx (SM)

${ }^{*}$ Corresponding author
}

Introduction: In Mexico, the state of Oaxaca is the second with higher mammalian richness. However, habitat loss and poaching have resulted in the decline and extinction of several species of flora and fauna. The present study focused on identifying the species richness and the relative abundance of medium and large mammals in the community of San Gabriel Casa Blanca, in the Biosphere Reserve Tehuacán-Cuicatlán. The work is important for the management and conservation of wildlife.

Methods: We placed 10 camera-traps during the dry season (November to April) and rainy season (May to October) in 2012. The camera traps were separated at least $500 \mathrm{~m}$ and were programmed to function $24 \mathrm{~h}$ a day, it took series of three photos at 10 to 15 second intervals. The cameras were reviewed every month. The richness and relative abundance during the dry and rainy season were estimated.

Results: An effort sampling of 3,300 night-trap was performed, and 826 independent records of 15 species of medium and large mammals were obtained. Additionally, Puma concolor was recorded by tracks. Only one species is under some risk category according to NOM-059-SEMARNAT-2010, Herpailurus yagouaroundi. The most abundant species were: Sylvilagus floridanus, Odocoileus virginianus, Urocyon cinereoargenteus and Dicotyles angulatus; while those of lower abundance were: Bassariscus astutus and Herpailurus yagouaroundi.

Discussion and conclusions: The results indicated that the species richness of medium and large mammals is similar to other areas with similar vegetation. In contrast, it is lower compared to other localities dominated by high and medium forests in Oaxaca.

Key words: Abundance; conservation; mammals; Oaxaca; richness; UMA. 


\section{Introducción}

Oaxaca es uno de los estados con mayor diversidad biológica en México por encontrarse en la zona de transición entre dos regiones biogeográficas: la Neártica y la Neotropical. Esto contribuye a su invaluable riqueza natural y ejemplo de ello son las 222 especies de mamíferos terrestres con los que cuenta (Santos-Moreno 2014). Sin embargo, siguen existiendo vacíos de información biológica para la mayoría de ellas (Briones-Salas 2000). En la Reserva de la Biosfera de TehuacánCuicatlán (RBTC), se han realizado esfuerzos encaminados a incrementar el conocimiento de las poblaciones y comunidades animales (v. gr. Briones-Salas 2000; Botello et al. 2006a; Ortíz-García y Mandujano 2011; Ortíz-García et al. 2012; Yáñez-Arenas et al. 2014). Particularmente, en la RBTC se ha empleado el foto-trampeo para documentar la diversidad de carnívoros y el patrón de actividad del venado cola blanca en la Región de La Cañada (Botello et al. 2005, 2006a, b, 2013; López-Tello 2014).

El foto-trampeo o de cámaras-trampa es un método que se aplica para obtener información de la presencia de los animales (O'Connell et al. 2011; Royle et al. 2014). En particular, esta técnica es muy útil en hábitats tropicales donde la detección de los mamíferos es complicada debido a factores como su baja abundancia, comportamiento evasivo, visibilidad en baja cobertura, terreno irregular y actividades humanas (Karanth et al. 2004). En consecuencia, el monitoreo de mamíferos entre diferentes localidades y épocas del año ha sido el foco principal de muchos estudios que emplean el foto-trampeo (Lyra-Jorge et al. 2008; Jenks et al. 2011; Gray 2012; Ahumada et al. 2013).

El presente trabajo se enfocó en identificar la riqueza y estimar la abundancia relativa de especies de mamíferos medianos y grandes en los Bienes Comunales de San Gabriel Casa Blanca, Oaxaca, en la RBTC, utilizando el método de foto-trampeo. El trabajo tiene importancia para el manejo y la conservación de fauna silvestre en la localidad de estudio, ya que actualmente es una Unidad para la conservación, manejo y aprovechamiento sustentable de la vida silvestre (UMA), del venado cola blanca y de otras especies de flora y fauna.

\section{Material y Métodos}

Área de estudio. Los bienes comunales de San Gabriel Casa Blanca (SGCB), municipio San Antonio Nanahuatipam, Oaxaca, se localizan en la región de La Cañada, en la Mixteca Oaxaqueña, y se enclava en el oriente-centro de la RBTC, en el extremo SE del estado de Puebla y el NE de Oaxaca ( $18^{\circ} 08^{\prime} 31^{\prime \prime} \mathrm{N},-97^{\circ} 07^{\prime} 46^{\prime \prime} \mathrm{W}$; Figura 1). Su altitud es poco variable (760 a $800 \mathrm{msnm}$ ), con una topografía dominada por terrenos accidentados entre cañadas, lomeríos, grietas y cerros (INEGI 2005; Barrera 2012). El clima es caluroso-semidesértico, con una temperatura media anual de 24 ${ }^{\circ} \mathrm{C}$ y una precipitación promedio de $438 \mathrm{~mm}$ con lluvias en verano y vientos del este en febrero y marzo. La tenencia de la tierra se distribuye en bienes comunales, ejido y pequeña propiedad (INEGI 2005). La UMA, con 2,000 ha decretadas, se localiza en la zona de transición entre dos tipos de vegetación: una de origen neártico (matorral crausicaule) y otra de origen neotropical (selva baja caducifolia), al igual que algunos manchones de pastizal inducido y zonas de cultivos (Valiente-Banuet et al. 2000; Dávila et al. 2002).

Colecta de datos. Se colocaron 10 cámaras-trampa digitales con sensor de movimiento (Primos Truth Cam $35^{\circledR}$ y Moultrie Game Spy D-55IR ${ }^{\circledast}$ ) de febrero a diciembre de 2012. Se abarcó la época de lluvias (mayo a octubre) y de secas (noviembre a abril). Las cámaras-trampa fueron colocadas en sitios donde se localizaron rastros (huellas, excretas, senderos, rascaderos), y en lugares sugeridos por guías locales, con una separación mínima entre ellas de $500 \mathrm{~m}$, de acuerdo con lo recomendado por otros autores (v. gr., Gutiérrez-González et al. 2012). Se sujetaron a troncos a 
una altura de 20 a $30 \mathrm{~cm}$ del suelo, con una orientación sur a norte para evitar la interferencia de rayos solares. Una vez instaladas, se georreferenciaron con la ayuda de un GPS Garmin eTrex ${ }^{\circledR}$ en unidades métricas (UTM). Se programaron para registrar eventos fotográficos de tres tomas consecutivas con intervalos de 10 a 15 segundos durante 24 horas y se revisaron mensualmente. Con los datos colectados, se conformó una base de datos y se ordenó la información de acuerdo con las coordenadas UTM, la clave de cámara, el nombre común y el científico de la especie fotografiada, la fecha (día/mes/año), la hora, fase lunar, número de individuos, sexo (cuando fue posible identificarlo), clave de la fotografía y observaciones generales. Se utilizó bibliografía especializada para la identificación de las especies (Hall 1981; Reid 1997; Ceballos y Oliva 2005; González-Christen 2010), así como el juicio de expertos para verificar registros dudosos. El arreglo taxonómico de las especies, así como su nombre científico, se basó en el trabajo de RamírezPulido et al. (2014).

Riqueza y abundancia de especies. Se estimó la riqueza específica y el índice de abundancia relativa (IAR) para cada especie y por época de lluvias y secas (Goulart et al. 2009). Las estimaciones de abundancia por especie se obtuvieron como resultado del conteo de los individuos capturados por la unidad de esfuerzo, calculando una fracción de la población con el número de registros independientes, diferenciados por ciclos de 24 horas. Se asume que todas las especies tienen la misma probabilidad de ser registradas (Aranda et al. 2012). El esfuerzo de muestreo se calculó sumando todos los días en que cada una de las cámaras permaneció activa. Se tomaron, como registros independientes, las fotografías consecutivas de individuos de diferentes especies, las

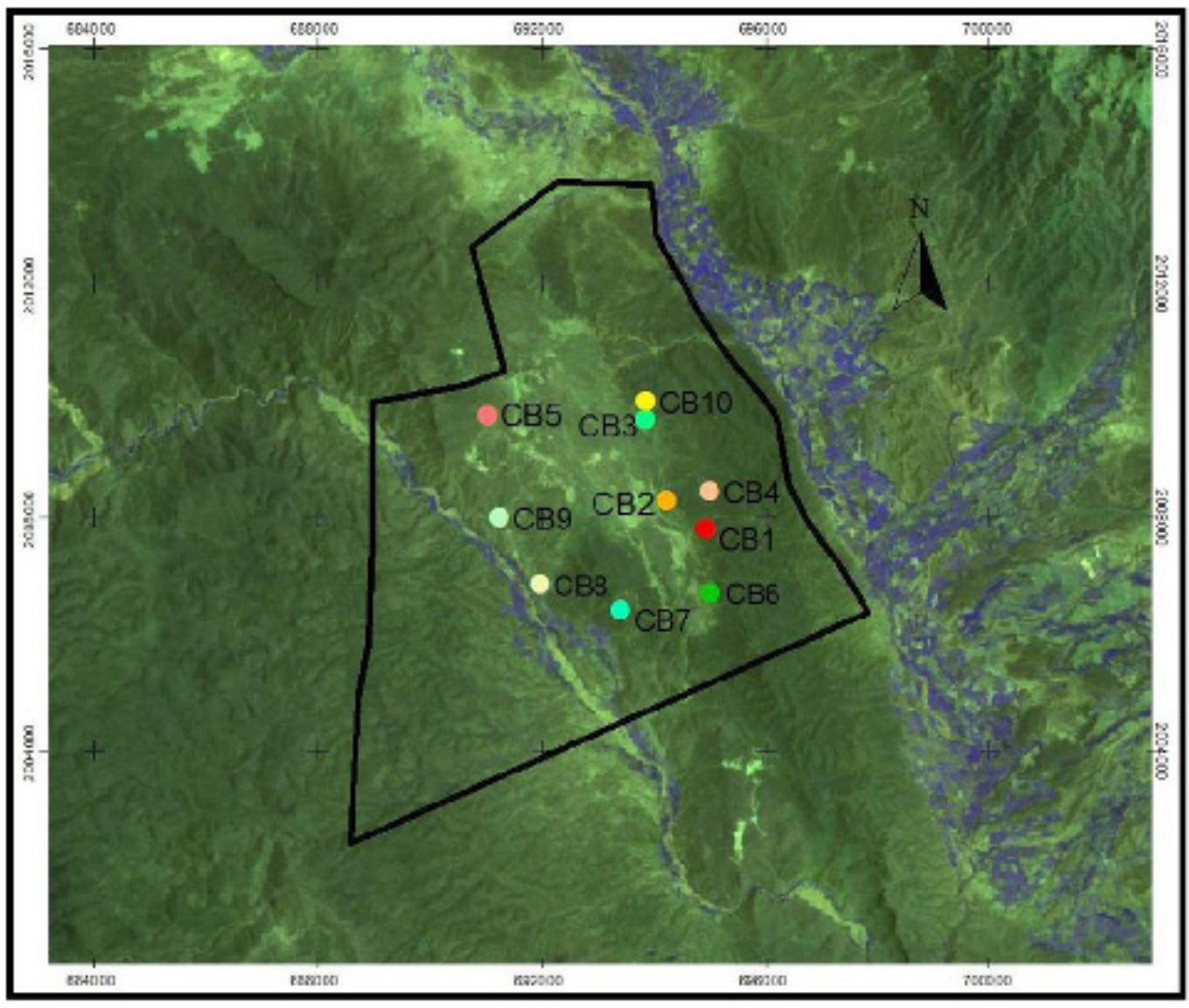

5

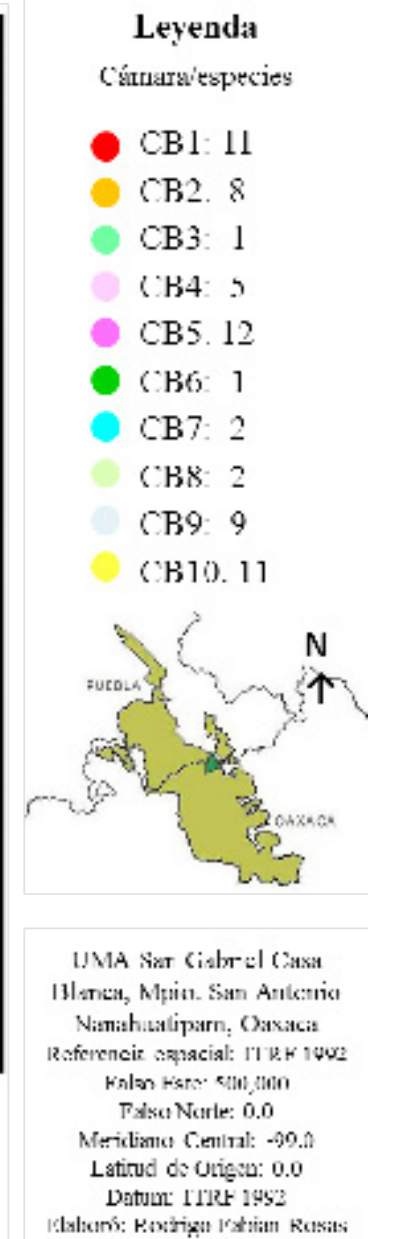

Figura 1. Localización geográfica del sitio de estudio, ubicado dentro de la Reserva de la Biosfera de Tehuacán-Cuicatlán, Oaxaca, y distribución de las cámaras y número de especies registradas en cada una. 
fotografías consecutivas de individuos de la misma especie separadas por más de 24 horas y las fotografías no consecutivas de individuos de la misma especie. En las fotografías en que se identificaron varios individuos, los registros se tomaron como el total de individuos capturados (Rowcliffe et al. 2008). Para obtener el IAR de cada especie, se utilizó la fórmula propuesta por Maffei et al. (2002) y Jenks et al. (2011): IAR = (C/EM) X 100. Donde: $C=$ número de capturas o eventos fotografiados, EM = esfuerzo de muestreo (número de cámaras por días de monitoreo) por época o total y 100 días-trampa (factor de corrección estándar).

Se compararon los valores del IAR para todo el conjunto de especies entre las dos épocas, empleando la prueba de $t$-Student para observaciones independientes con nivel de significancia de $P \leq 0.05$ (Tabla 1). Por otro lado, con el fin de determinar probables diferencias significativas en el IAR para cada una de las especies por épocas, se aplicó una prueba binomial de comparación de igualdad de proporciones con la corrección para la continuidad de datos (Crawley 2013). En este caso, se empleó el número de observaciones independientes de la especie en función del número de días de foto-trampeo para cada una de las dos épocas. La prueba evalúa la similitud proporcional entre épocas. En caso de valor significativo, se concluye que el IAR varía entre épocas para la misma especie. Para este caso, se empleó la función prop.test en el programa $\mathrm{R}$ vr. 3.1.3 (R Core Team 2015).

\section{Resultados}

Se obtuvo un total de 826 registros independientes de 3,459 fotografías, con un esfuerzo total de muestreo de 3,300 cámaras-día. Los datos obtenidos con foto-trampeo y con métodos indirectos (huellas) para ambas épocas del año revelaron una riqueza específica de 16 especies compuestas por cinco órdenes (Didelphimorphia, Cingulata, Lagomorpha, Carnivora y Artiodactyla), nueve familias y 16 géneros. Los registros obtenidos con el foto-trampeo muestran que solo en cuatro familias (Leporidae, Cervidae, Canidae y Tayassuidae) se concentra $87 \%$ de los registros obtenidos únicamente en la época de lluvias (Figura 2). En secas se mantiene un patrón similar, aunque la familia Tayassuidae se sustituye por la familia Mephitidae (Figura 2), ya que los pecaríes de collar no fueron registrados en la época de secas.

De las especies registradas, 11 pertenecen al Orden Carnivora y están distribuidas en cuatro familias. Las más representadas fueron Felidae, Mephitidae y Procyonidae, con tres especies cada una (Tabla 1, Figura 3 y 4). Es importante mencionar que Puma concolor fue registrada por medio de huellas durante los recorridos. Las 15 especies restantes fueron registradas con el fototrampeo. De las 16 registradas, solo Herpailurus yagouaroundi se encuentra catalogada en la NOM-059-SEMARNAT-2010 (SEMARNAT 2010) como especie amenazada (A).

Las especies con mayor frecuencia de foto-captura para ambas épocas fueron: Sylvilagus floridanus ( $n=321$ registros, $38.40 \%$ ), Odocoileus virginianus ( $n=263$ registros, $31.46 \%$ ) y Urocyon cinereoargenteus ( $n=93$ registros, $11.12 \%$ ). En cambio, las especies con menor frecuencia fueron: Bassariscus astutus y $\mathrm{H}$. yagouaroundi (Tabla 1, Figura 4). En cuanto a los registros por épocas, en Iluvias se registraron 15 especies, mientras que en la época seca, 12 especies. No se registraron $H$. yagouaroundi, B. astutus y Didelphis virginiana (Figura 4).

Las especies con mayor abundancia relativa estimada $(\bar{X})$ en ambas épocas fueron ( \pm D.E.): el conejo, S. floridanus $\left(\bar{X}_{I A R}=10.01 \pm 0.22\right)$ y el venado cola blanca, O. virginianus $\left(\bar{X}_{I A R}=8.13 \pm 0.88\right)$, seguidas de la zorra gris, $U$. cinereoargenteus con una abundancia relativa cuatro veces menor

que los venados $\left(\bar{X}_{\text {IAR }}=2.94 \pm 0.31\right)$. El resto de las especies $(n=12)$ con un $\overline{\mathrm{X}}_{\text {IAR }}<1.16$ para ambas épocas. Las especies menos abundantes fueron el cacomixtle, $B$. astutus y el jaguarundi, $H$. yagouaroundi $\left(\overline{\mathrm{X}}_{I A R}=0.03 \pm 0.04\right.$ ambas especies, respectivamente; Tabla 1, Figura 4). Por otro lado, es importante notar que especies consideradas como muy comunes y con altas probabilidades 
Tabla 1. Comparación del número de registros independientes del foto-trampeo e índice de abundancia relativa (IAR) por especie y época. El orden taxonómico y los nombres científicos de las especies es el propuesto por Ramírez-Pulido et al. (2014). No se incluye a P. concolor debido a que solo fue registrado por rastros. Prueba $X^{2}$ binomial prueba binomial de comparación de igualdad de proporciones.

\begin{tabular}{|c|c|c|c|c|c|c|c|c|c|}
\hline \multirow{2}{*}{ Orden } & \multirow{2}{*}{ Familia } & \multirow{2}{*}{ Especie } & \multicolumn{2}{|c|}{ Registros } & \multicolumn{2}{|c|}{ IAR } & \multirow{2}{*}{$\bar{X}_{(I A R j+D . E .)}$} & \multirow{2}{*}{$X^{2}$} & \multirow{2}{*}{$P$} \\
\hline & & & Lluvia & Seca & Lluvia & Seca & & & \\
\hline Didelphimorphia & Didelphidae & Didelphis virginiana Kerr, 1792 & 2 & 0 & 0.11 & 0.00 & $0.05 \pm 0.08$ & 0.252 & 0.62 \\
\hline Cingulata & Dasypodidae & $\begin{array}{l}\text { Dasypus novemcinctus } \\
\text { Linnaeus, } 1758\end{array}$ & 2 & 1 & 0.11 & 0.07 & $0.09 \pm 0.02$ & 0.041 & 0.84 \\
\hline \multirow[t]{6}{*}{ Lagomorpha } & Leporidae & $\begin{array}{l}\text { Sylvilagus floridanus (J. A. } \\
\text { Allen, 1890) }\end{array}$ & 187 & 134 & 10.16 & 9.85 & $10.01 \pm 0.22$ & 0.869 & 0.35 \\
\hline & & Lynx rufus (Schreber, 1777) & 22 & 14 & 1.20 & 1.03 & $1.11 \pm 0.12$ & 0.082 & 0.75 \\
\hline & Felidae & $\begin{array}{l}\text { Herpailurus yagouaroundi (È. } \\
\text { Geoffroy Saint-Hilaire, 1803) }\end{array}$ & 1 & 0 & 0.05 & 0.00 & $0.03 \pm 0.04$ & \multicolumn{2}{|c|}{ Datos insuficientes } \\
\hline & & Canis latrans Say, 1822 & 5 & 2 & 0.27 & 0.15 & $0.21 \pm 0.09$ & 0.135 & 0.71 \\
\hline & Canidae & $\begin{array}{l}\text { Urocyon cinereoargenteus } \\
\text { (Schreber, 1775) }\end{array}$ & 50 & 43 & 2.72 & 3.16 & $2.94 \pm 0.31$ & 0.549 & 0.46 \\
\hline & \multirow[b]{3}{*}{ Mephitidae } & $\begin{array}{l}\text { Conepatus leuconotus } \\
\text { (Lichtenstein, 1832) }\end{array}$ & 14 & 8 & 0.76 & 0.59 & $0.67 \pm 0.12$ & 0.144 & 0.74 \\
\hline \multicolumn{10}{|l|}{ Carnivora } \\
\hline & & $\begin{array}{l}\text { Mephitis macroura } \\
\text { Lichtenstein, } 1832\end{array}$ & 2 & 3 & 0.11 & 0.22 & $0.16 \pm 0.08$ & 0.117 & 0.73 \\
\hline & & $\begin{array}{l}\text { Spilogale angustifrons A. H. } \\
\text { Howell, } 1902\end{array}$ & 10 & 14 & 0.54 & 0.29 & $0.42 \pm 0.18$ & 2.01 & 0.16 \\
\hline & \multirow{3}{*}{ Procyonidae } & $\begin{array}{l}\text { Bassariscus astutus } \\
\text { (Lichtenstein, 1830) }\end{array}$ & 1 & 0 & 0.05 & 0.00 & $0.03 \pm 0.04$ & \multicolumn{2}{|c|}{ Datos insuficientes } \\
\hline & & Nasua narica (Linnaeus, 1766) & 8 & 6 & 0.43 & 0.44 & $0.44 \pm 0$ & 0.005 & 0.95 \\
\hline & & Procyon lotor (Linnaeus, 1758) & 1 & 1 & 0.05 & 0.07 & $0.06 \pm 0.01$ & \multicolumn{2}{|c|}{ Datos insuficientes } \\
\hline \multirow{2}{*}{ Artiodactyla } & Tayassuidae & $\begin{array}{l}\text { Dicotyles angulatus Cope, } \\
1889\end{array}$ & 40 & 2 & 2.17 & 0.15 & $1.16 \pm 1.43$ & 26.43 & 0.0001 \\
\hline & Cervidae & $\begin{array}{l}\text { Odocoileus virginianus } \\
\text { (Zimmermann, 1780) }\end{array}$ & 161 & 102 & 8.75 & 7.50 & $8.13 \pm 0.88$ & 8.334 & 0.004 \\
\hline
\end{tabular}

de ser registradas, en SGCB tuvieron muy pocos registros $y$, por ende, abundancias relativas con valores bajos, como Procyon lotor, D. virginiana, Dasypus novemcinctus, Mephitis macroura y Canis latrans (Figura 4).

En cuanto a las abundancias relativas por especie y época, destacan los resultados obtenidos para el pecarí de collar (Dicotyles angulatus), que en época de lluvias fue más abundante que en secas (IAR $=2.17$ y 40 registros independientes, IAR $=0.15$ y 2 registros independientes, respectivamente). Las abundancias relativas fueron estadísticamente similares entre la época de lluvias y la de secas ( $t$ $=0.689, \mathrm{~g} . \mathrm{l} .=14, P=0.49$ ). No obstante, el análisis estadístico con la separación de cada una de las 


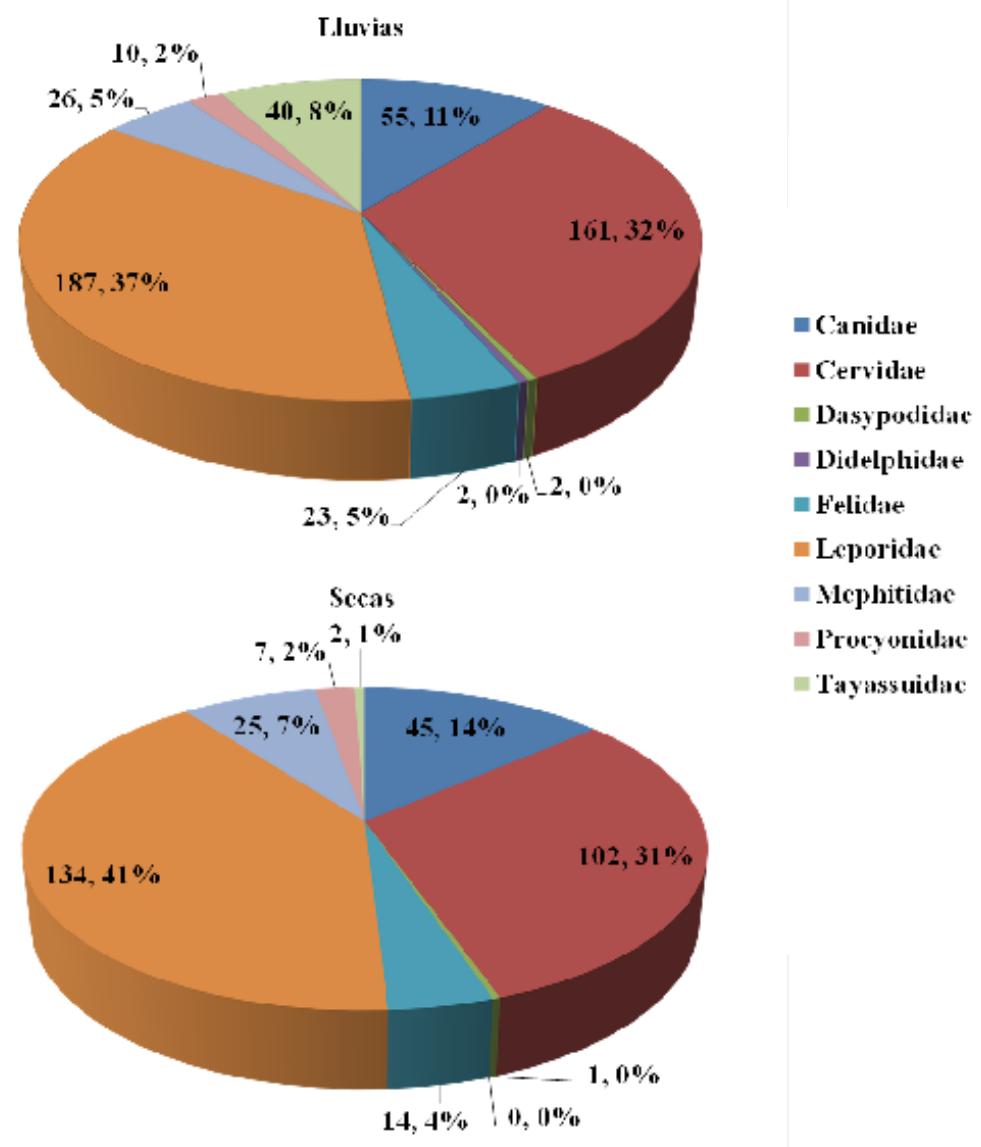

Figura 2. Representación proporcional de los registros obtenidos por familia y épocas con la técnica de foto-trampeo en San Gabriel Casa Blanca, Oaxaca.

especies por época arrojó que existen diferencias significativas entre las abundancias estimadas solo para dos especies $(P<0.05)$ : el pecarí de collar $\left(D\right.$. angulatus, $\left.X^{2}=26.43, \mathrm{~g} . \mathrm{I} .=1, P=0.0001\right)$ y el venado cola blanca (O. virginianus, $X^{2}=8.334$, g. I. $=1, P=0.004$; Tabla 1 ). El resto de las especies no tuvo diferencias significativas en las estimaciones de abundancia entre épocas, es decir, el patrón de los resultados se muestra similar tanto para lluvias como para secas.

\section{Discusión}

Las especies de mamíferos medianos y grandes en el área de estudio ya habían sido registradas anteriormente para la RBTC (Briones-Salas 2000; Botello et al. 2006 a, b, 2013). Sin embargo, es importante resaltar que SGCB, enclavado en la Región de La Cañada, alberga al $26 \%$ de los carnívoros registrados en el país. Este gremio trófico es el más ampliamente registrado en el área. Aunado a esto, destacan la presencia de cuatro de las seis especies de felinos mexicanos, lo que representa $2 / 3$ de las especies de felinos del país. La mayor importancia de la presente contribución radica en la diferencia sustancial entre los resultados aquí reportados y los reportados con anterioridad. Si bien en 2000 se publicó un listado de los mamíferos de la Región de la Cañada y se reportan 26 especies, solo 10 de ellas se consideran mamíferos de talla mediana a grande (Briones-Salas 2000). Posteriormente, se reportaron Leopardus wiedii, Spilogale angustifrons y $\mathrm{H}$. yagouaroundi (Botello et al. 2006 a, b, 2013). En este estudio, registramos cuatro especies de mamíferos medianos y grandes que no habían sido reportadas previamente en la Región de La Cañada (Briones-Salas 2000; Botello et al. 2006 a, b, 2013): S. floridanus, Lynx rufus, C. latrans y $D$. angulatus, consideradas todas especies comunes. En particular, tomando en cuenta 
todas las tallas de mamíferos terrestres, la localidad de estudio tiene $60 \%$ de las especies registradas en la región, y 6.8 \% en el estado de Oaxaca (Cervantes y Riveros 2012; Santos-Moreno 2014).

Los resultados muestran que la riqueza de mamíferos medianos y grandes de Casa Blanca es similar a lo obtenido en otras áreas del estado de Oaxaca; por ejemplo, en sitios con tipos de

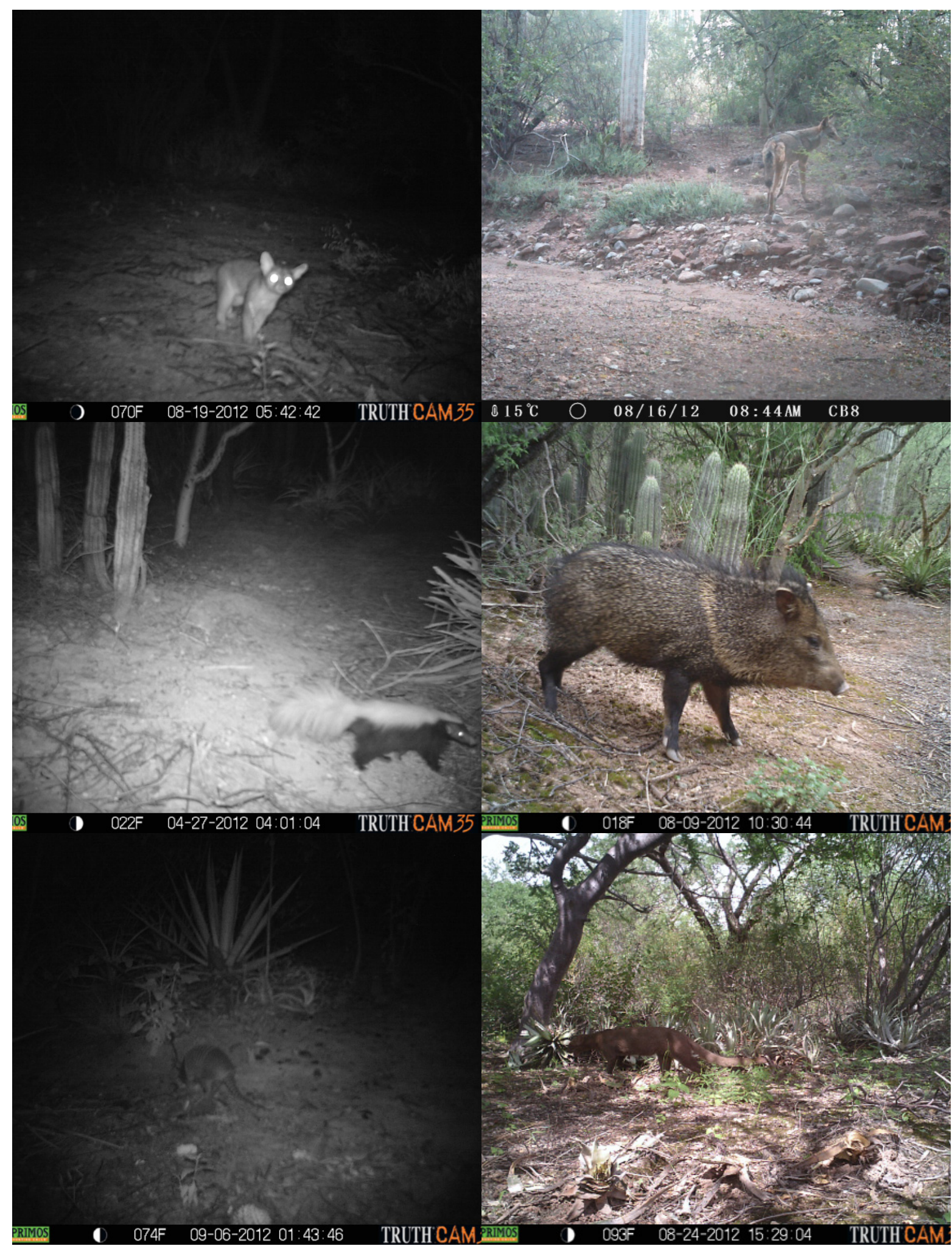

Figura 3. Imágenes de algunas de las especies capturadas con foto-trampeo en San Gabriel Casa Blanca, Oaxaca. De izquierda a derecha: $B$. astutus, C. latrans, C. leuconotus, D. angulatus, D. novemcinctus y $H$. yagoarundi. 
vegetación similar (selva baja caducifolia) al área de estudio, se han reportado entre 11 y 15 especies de mamíferos de talla mediana (Alfaro et al. 2006; Cervantes y Riveros 2012; Pérez-Irineo y Santos-Moreno 2012). En este estudio, se reportan 16 especies. Sin embargo, la riqueza de

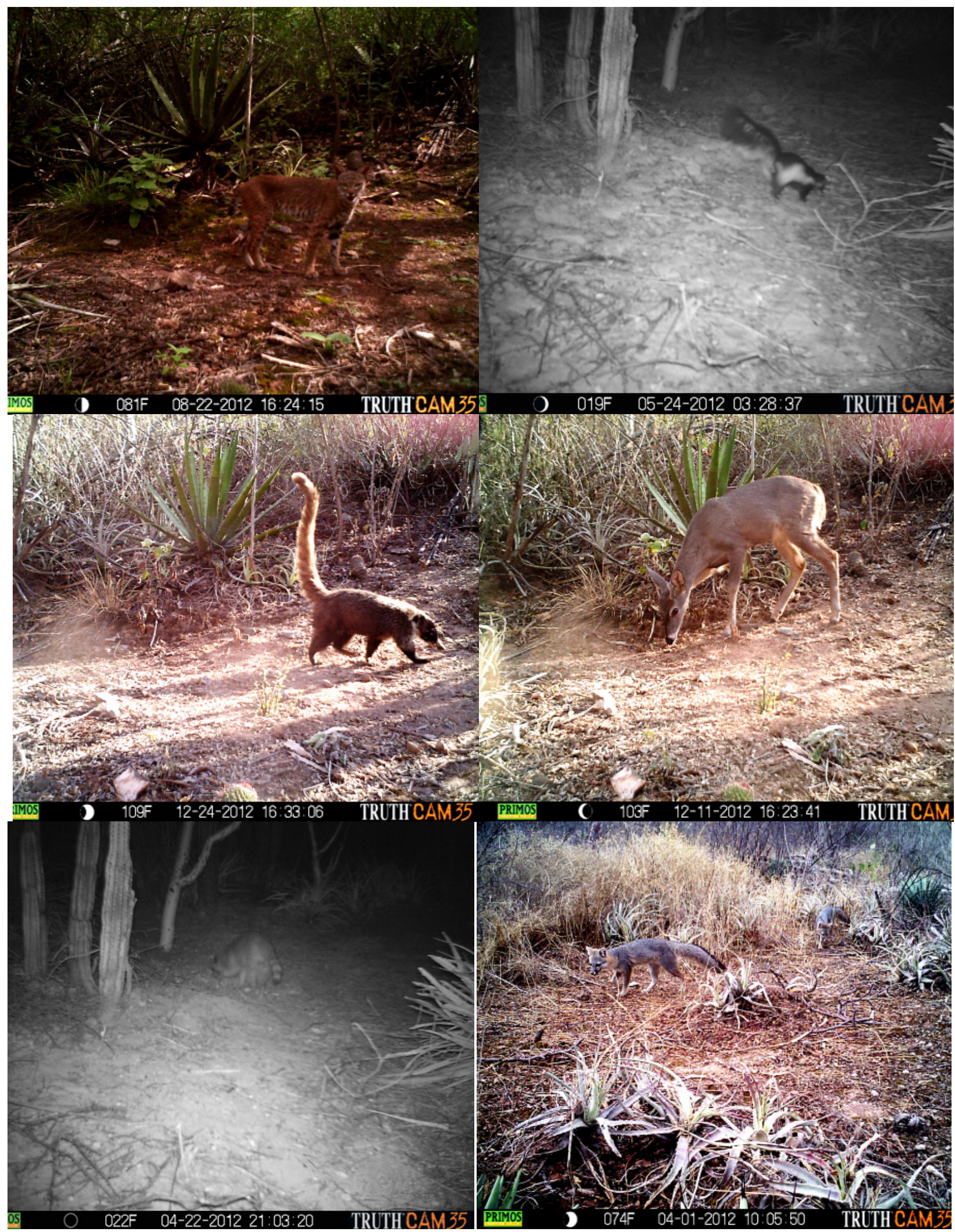

Figura 3. Imágenes de algunas de las especies capturadas con foto-trampeo en San Gabriel Casa Blanca, Oaxaca. De izquierda a derecha: L. rufus, M. macroura, N. narica, O. virginianus, P. lotor y U. cinereoargenteus. 
especies de tallas mediana a grande obtenidas en la presente contribución es sustancialmente menor a lo registrado en la Región Los Chimalapas (Carnivora 17, Didelphimorphia 6, Artiodactyla 4, Pilosa 2, Lagomorpha 2, Cingulata 1, y Perissodactyla 1; Olguín-Monroy et al. 2008). Aun cuando se muestran en selva baja caducifolia, esta diferencia probablemente se deba a la localización latitudinal de Los Chimalapas (mucho más al sur-sureste que SGCB), situación biogeográfica que apunta a que, en gran medida, la riqueza específica de mamíferos aumenta conforme disminuye el gradiente latitudinal (Willing et al. 2003; Olguín-Monroy et al. 2008).

Las especies más abundantes para ambas épocas del año fueron: S. floridanus, O. virginianus, $U$. cinereoargenteus y $D$. angulatus. En el caso del venado cola blanca, los resultados del presente estudio (a pesar de que no se realizaron estimaciones de densidad) son consistentes con lo reportado para Casa Blanca, donde se estimaron densidades relativamente altas (Ramos-Robles et al. 2013) y pueden estar influidas por la disponibilidad de recursos alimenticios y de refugio. La tercera especie más abundante fue $U$. cinereoargenteus. Lo anterior contrasta con estudios realizados en Los Chimalapas, donde fue de las menos abundantes (Olguín-Monroy et al. 2008). En cuarto lugar resultó el pecarí de collar ( $D$. angulatus), que en otros estudios resulta ser muy abundante (Olguín-Monroy et al. 2008). La abundancia notable de estas cuatro especies, particularmente de los herbívoros, puede explicarse por la disponibilidad de los recursos (alimento, refugio) que mantiene la UMA SGCB. Dichos recursos se relacionan con el tipo de vegetación dominante de la región de La Cañada (bosque tropical caducifolio), el cual proporciona una mejor disponibilidad de cobertura arbórea y arbustiva, así como protección contra radiación solar y depredación. En cuanto a las abundancias del

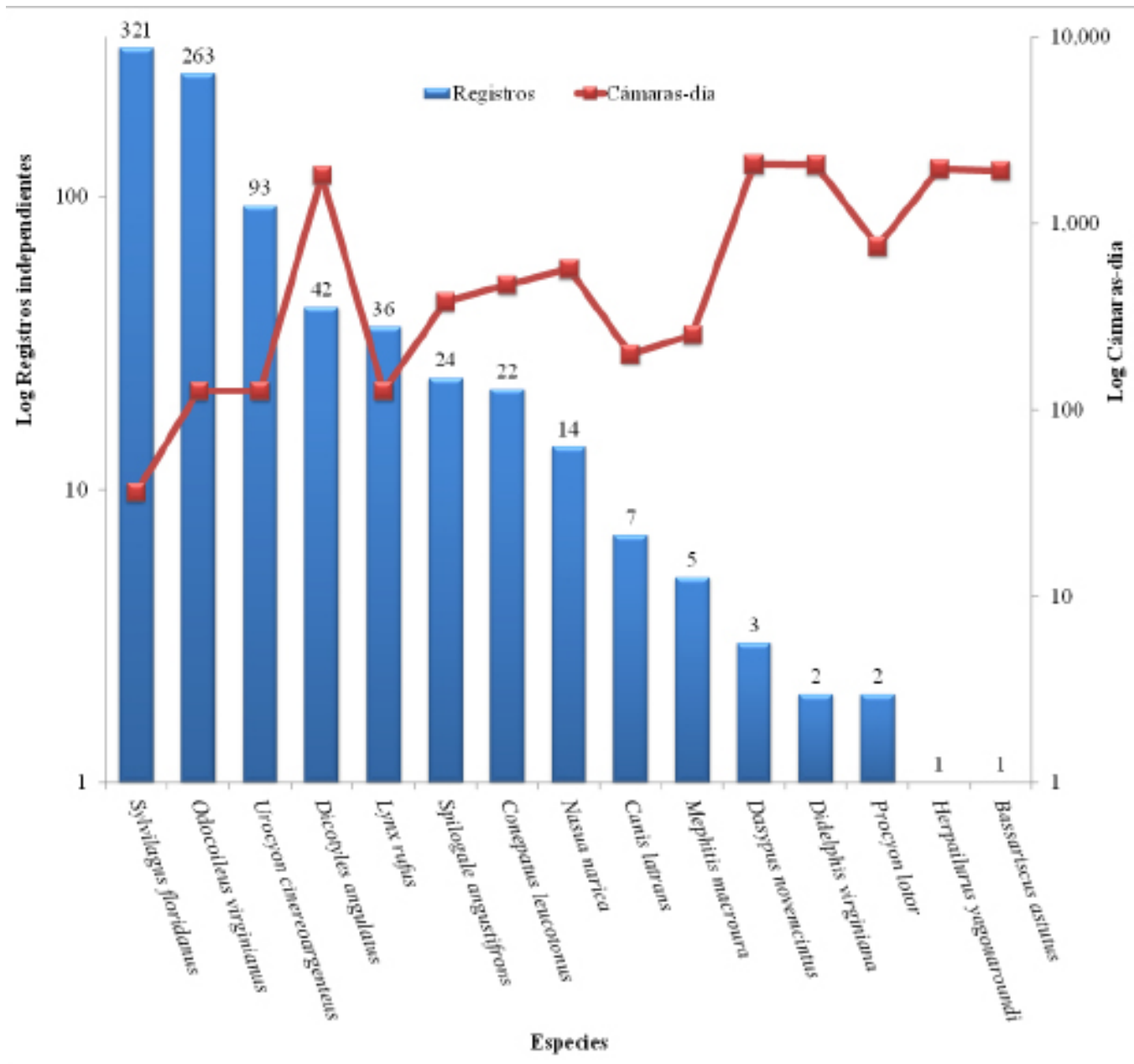

Figura 4. Registros independientes de las especies fotografiadas en San Gabriel Casa Blanca en ambas épocas. No se incluye a $P$. concolor porque solo fue registrado por rastros durante los muestreos en campo. 
venado y del pecarí de collar, es probable que sean reflejo del estado de conservación que guarda la vegetación en la UMA, a diferencia de otros sitios en la misma región. Estas características presentes en la UMA SGCB, aunadas a que se trata de un área en constante vigilancia comunitaria por sus pobladores, le confieren a la zona un importante valor como sitio de conservación de la diversidad biológica de la RBTC.

Las especies menos abundantes fueron $P$. lotor, D. novemcinctus, B. astutus y $H$. yagouaroundi. El dominante es el Orden Carnívora, con 11 especies de las 16 registradas. No obstante, la especie más abundante resulta ser un herbívoro (S. floridanus), pieza importante en la dieta de al menos cuatro de las especies de carnívoros registrados ( $P$. concolor, $H$. yagouaroundi, $C$. latrans y L. rufus).

Resulta interesante que para el caso de las especies consideradas medianamente abundantes (33\% de las especies), la lista esté dominada por el Orden Carnivora, con seis especies ( $M$. macroura, C. latrans, Nasua narica, S. angustifrons, Conepatus leuconotus y L. rufus). De ellas, el lince (L. rufus) y el zorrillo cadeno (C. leuconotus) fueron las más frecuentemente fotografiadas, aun cuando se podría esperar que por sus hábitos evasivos, el lince fuera menos frecuente y abundante; no obstante, resultó lo contrario. El fenómeno anterior puede relacionarse con la abundancia de presas como los conejos, en particular durante la temporada de lluvias.

El coyote (C. latrans) es otra especie que abunda en muchos lugares del país. Sin embargo, los resultados aquí obtenidos no lo muestran. Esto puede estar supeditado a la presión por cacería, a la que la especie está sujeta históricamente, aun cuando se localice dentro de un área natural protegida. Por último, las especies menos abundantes fueron $B$. astutus y $H$. yagouaroundi (especie amenaza). Este fenómeno puede deberse a que son especies difíciles de observar en campo por sus hábitos sigilosos y de evasión, propios de los carnívoros. Finalmente, es importante considerar que la riqueza de especies registradas en el área puede estar influida por el hecho de que SGCB posee un registro como UMA, por lo que se realizan actividades de protección y vigilancia, lo que evidencia que este tipo de estrategias es una alternativa para la conservación y el mantenimiento de la biodiversidad.

\section{Agradecimientos}

Agradecemos a E. Suárez Domínguez, J. Benítez Rodríguez, Ma. A. Chamorro y G. Castro Bobadilla por los comentarios al trabajo de tesis del primer autor, a dos revisores anónimos del manuscrito y al editor asociado, C. Lorenzo, por sus acertadas observaciones y recomendaciones. Para realizar el trabajo, se contó con el apoyo durante los muestreos y con el apoyo logístico de la Red de vigilancia de SGCB, Red de Biología y Conservación de Vertebrados del Instituto de Ecología A. C. (INECOL, A. C.), Laboratorio de Zoología del Instituto de Investigaciones Biológicas-UV, de R. Fabian y del A. González-Christen. El presente trabajo fue financiado por CONACyT a través del INECOL, A. C. con el proyecto CB-2009-01-130702 “Evaluación de las interacciones entre el venado cola blanca y el ganado en la reserva de la biosfera Tehuacán-Cuicatlán: implicaciones de conservación y manejo" otorgado a S. Mandujano y parcialmente por el Patronato ProUniversidad Veracruzana A. C.

\section{Resumen}

Introducción: En México, el estado de Oaxaca ocupa el segundo lugar en riqueza de mamíferos; sin embargo, la pérdida de hábitat y la caza furtiva han dado lugar a la disminución y extinción de varias especies de flora y fauna. El presente estudio se centró en determinar la riqueza de especies y la abundancia relativa de mamíferos de talla mediana y grande en la comunidad de San Gabriel Casa Blanca, Oaxaca, en la Reserva de la Biosfera TehuacánCuicatlán. Este estudio es importante para el manejo y conservación de la vida silvestre. 
Material y métodos: Métodos: Se colocaron 10 cámaras trampa, que permanecieron activas de febrero a diciembre de 2012, durante la época de lluvias (mayo a octubre) y de secas (noviembre a abril). La separación entre una y otra cámara fue de al menos $500 \mathrm{~m}$ lineales. Se programaron para estar activas las 24 horas, tomando series de tres fotos a intervalos de 10 a15 segundos y se revisaron cada mes. Se estimó la riqueza específica y la abundancia relativa de cada especie y por época.

Resultados: Se realizó un esfuerzo de muestreo de 3,300 días-trampa. Se obtuvieron 826 registros independientes de 15 especies de mamíferos de talla mediana a grande. Adicionalmente, se registró al Puma concolor por medio de huellas. Solo una especie se encuentra bajo categoría de riesgo en la NOM-059-SEMARNAT-2010: Herpailurus yagouaroundi. Las especies con mayor abundancia relativa fueron Sylvilagus floridanus, Odocoileus virginianus, Urocyon cinereoargenteus y Dicotyles angulatus. Las de menor abundancia fueron Bassariscus astutus y Herpailurus yagouaroundi.

Discusión y conclusiones: Los resultados indicaron que la riqueza de especies de mamíferos medianos y grandes es similar a otras áreas con vegetación parecida. Por el contrario, es más baja en comparación con otras localidades dominadas por bosques tropicales en Oaxaca.

Palabras clave: Abundancia, conservación, mamíferos, Oaxaca, riqueza, UMA.

\section{Literatura citada}

Ahumada, J., J. Hurtado, y D. Lizcano. 2013. Monitoring the status and trends of tropical forest, terrestrial vertebrate communities from camera trap data: a tool for conservation. PLOS ONE 8:e73707.

Alfaro, A., L. García, y A. Santos-Moreno. 2006. Mamíferos de los municipios Santiago Jocotepec y Ayotzintepec, Chinantla Baja, Oaxaca. Revista Naturaleza y Desarrollo 4:19-23.

Aranda, M., F. Botello, y L. López-De Buen. 2012. Diversidad y datos reproductivos de mamíferos medianos y grandes en el bosque mesófilo de montaña de la Reserva de la Biosfera Sierra de Manantlán, JaliscoColima, México. Revista Mexicana de Biodiversidad 83:778-784.

BarRera, A. 2012. Caracterización y evaluación del hábitat del venado cola blanca (Odocoileus virginianus mexicanus) en los bienes comunales de San Gabriel Casa Blanca, San Antonio Nanahuatipam, Oaxaca. Tesis de licenciatura. Benemérita Universidad Autónoma de Puebla. Puebla, México.

Botello, F., P. Illoldi, M. Linaje, G. Monroy, y V. Sánchez-Cordero. 2005. Nuevos registros del tepezcuintle (Agouti paca) para el norte del estado de Oaxaca, México. Revista Mexicana de Biodiversidad 76:103-105.

Botello, F., P. Illoldi-Rangel, M. Linaje, y V. Sánchez-Cordero. 2006a. Primer registro del tigrillo (Leopardus wiedii, Schinz1821) y del gato montés (Lynx rufus, Kerr 1792) en la Reserva de la Biosfera de TehuacánCuicatlán, Oaxaca, México. Acta Zoológica Mexicana 22:135-139.

Botello, F., J. M. Salazar, P. Illoldi, M. Linaje, G. Monroy, D. Duque, y V. Sánchez-Cordero. 2006b. Primer registro de la nutria neotropical de río (Lontra longicaudis) en la Reserva de la Biosfera de TehuacánCuicatlán, Oaxaca, México. Revista Mexicana de Biodiversidad 77:133-135.

Botello, F., E. Villaseñor, L. Guevara, Á. Méndez, A. Cortés, J. Iglesias, M. Izúcar, M. Luna, A. Martínez, y J. M. Salazar. 2013. Registros notables del zorrillo manchado (Spilogale angustifrons) y del jaguarundi (Puma yagouaroundi) en la Reserva de la Biosfera de Tehuacán-Cuicatlán, Oaxaca, México. Revista Mexicana de Biodiversidad 84:713-717.

Briones-Salas, M. 2000. Lista anotada de los mamíferos de la región de La Cañada, en el valle de TehuacánCuicatlán, Oaxaca, México. Acta Zoológica Mexicana (n. s.) 81:83-103.

Ceballos, G., y G. Oliva. 2005. Los mamíferos silvestres de México. CONABIO/ Fondo de Cultura Económica. Ciudad de México, México.

Cervantes, F., y B. Riveros. 2012. Mamíferos del Municipio de Cosoltepec, Oaxaca, México. Therya 3:311-325.

Crawley, M. J. 2013. The R Book. Second edition. John Wiley and Sons, Reino Unido

Dávila, P., M. del Coro Arizmendi, A. Valiente-Banuet, J. L. Villaseñor, A. Casas, y R. Lira. 2002. Biological diversity in the Tehuacán-Cuicatlán Valley, Mexico. Biodiversity and Conservation 11:421-442.

González-Christen, A. 2010. Los Mamíferos de Veracruz: Guía llustrada. Primera edición. Colección La Ciencia en Veracruz. Consejo Veracruzano de Investigación Científica y Desarrollo Tecnológico-Secretaría de Educación Pública. Xalapa, México. 
Goulart, F. V. B., N. C. Caceres, M. E. Graipel, M. A. Tortato, I. R. Ghizoni, y L. R. Oliveira-Santos. 2009. Habitat selection by large mammals in a southern Brazilian Atlantic Forest. Mammalian Biology 74:184-192.

GRAY, T. N. E. 2012. Studying large mammals with imperfect detection: status and habitat preferences of wild cattle and large carnivores in eastern Cambodia. Biotropica 44:531-536.

Gutiérrez-González, C. E., M. A. Gómez-Ramírez, y C. A. López-González. 2012. Estimation of the density of the near threatened jaguar Panthera onca in Sonora, Mexico, using camera trapping and an open population model. Oryx 46:431-437.

HALL, R. 1981. The mammals of North American. Willey-Interscience. New York, EE. UU.

ineGi (Instituto Nacional De Estadística, Geografía e Informática). 2005. Prontuario de información geográfica municipal de los Estados Unidos Mexicanos. San Antonio Nanahuatipam, Oaxaca. Ciudad de México, México.

Jenks, K. E., P. Chanteap, K. Damrongchainarong, P. Cutter, T. Redford, A. J. Lynam, J. Howard, y P. Leimgruber. 2011. Using relative abundance indices from camera-trapping to test wildlife conservation hypotheses - an example from Khao Yai National Park, Thailand. Tropical Conservation Science 4:113-131.

Karanth, K. U., J. D. Nichols, y N. S. Kumar. 2004. Photographic sampling of elusive mammals in tropical forests. Pp. 229-247 en Sampling rare or elusive species: concepts, designs and techniques for estimating population parameters (Thompson, E. L., ed.). Island Press. Washington, EE.UU.

López-Telzo, E. 2014. Patrón de actividad del venado cola blanca (Odocoileus virginianus) en la Reserva de la Biosfera Tehuacán-Cuicatlán. Tesis de Maestría. Instituto de Neuroetología, Universidad Veracruzana. Xalapa, México.

Lyra-Jorge, M. C., G. Ciocheti, V. R. Pivello, y S. T. Meirelles. 2008. Comparing methods for sampling large-and medium sized mammals: camera traps and track plots. European Journal of Wildlife Research 54:739-744.

Maffel, L., E. Cuellar, y J. Noss. 2002. Uso de trampas cámara para la evaluación de mamíferos en el ecotono Chaco-Chiquitanía. Revista Boliviana de ecología y conservación 11:55-65.

O'connell, A. F., J. D. Nichols, y K. U. Karanth (Eds.). 2011. Camera traps in animal ecology: methods and analyses, primera edición, Springer Press. New York, EE. UU.

Olguín-Monroy, H., L. León Paniagua, U. M. Samper-Palacios, y V. Sánchez-Cordero. 2008. Mastofauna de la región de los Chimalapas, Oaxaca, México. Pp. 165-216 in Avances en el estudio de los mamíferos de México, vol. II. (Lorenzo-Monterrubio, C., E. Espinoza-Medinilla, y J. Ortega, eds.) Publicaciones especiales. Asociación Mexicana de Mastozoología. Ciudad de México, México.

Ortíz-García, A. I., y S. Mandujano. 2011. Evaluación de la calidad del hábitat para el pecarí de collar en una Reserva de Biosfera de México. IUCN/SSC Wild Pig, Peccary, and Hippo Specialist Groups, Suiform Soundings 11:14-27.

Ortíz-García, A. I., M. I. Ramos-Robles, L. A. Pérez-Solano, y S. Mandujano. 2012. Distribución potencial de los ungulados silvestres en la Reserva de Biosfera de Tehuacán-Cuicatlán, México. Therya 3:333348.

Pérez-Irineo, G., y A. Santos-Moreno. 2012. Diversidad de mamíferos terrestres de talla grande y media de una selva subcaducifolia del noreste de Oaxaca, México. Revista Mexicana de Biodiversidad 83:164-169.

Ramírez-Pulido, J., N. González-Ruiz, A. Gardner, y J. Arroyo-Cabrales. 2014. List of recent land mammals of Mexico. Special Publications of the Museum of Texas Tech University 63:1-69.

Ramos-Robles, M. I, S. Gallina, y S. Mandujano. 2013. Habitat and human factors associated with whitetailed deer density in the tropical dry forest of Tehuacán-Cuicatlán Biosphere Reserve, Mexico. Tropical Conservation Science 6:70-86.

R Core Team. 2015. R: A language and environment for statistical computing. R Foundation for Statistical 
Computing, Vienna, Austria. URL http://www.R-project.org/.

Reid, A. F. 1997. A field guide to the mammals of Central America and Southeast Mexico. Oxford University Press. New York, E.E.U.U.

Rowcliffe, J., J. Field, S. T. Turvey, y C. Carbone. 2008. Estimating animal density using camera traps without the need for individual recognition. Journal of Applied Ecology 45:1228-1236.

Royle, J. A., R. B. Chandler, R. Sollmann, y B. Gardner. 2014. Spatial capture-recapture. Academic Press Elsevier Inc. Waltham, EE. UU.

Santos-Moreno, A. 2014. Los Mamíferos del estado de Oaxaca. Revista Mexicana de Mastozoología 4:18-32.

Semarnat (Secretaría de Medio Ambiente y Recursos Naturales). 2010. Norma Oficial Mexicana NOM-059SEMARNAT-2010, Protección Ambiental-Especies nativas de México de Flora y Fauna SilvestresCategorías de Riesgo y Especificaciones para su inclusión, exclusión o cambio-Lista de especies en riesgo. Diario Oficial, 56, 2a. Sección: 1-85.

Valiente-Banuet, A., A. Casas, A. Alcántara, P. Dávila, N. Flores Hernández, M. C. Arizmendi, J. L. Villaseñor, y J. Ortega. 2000. La vegetación del Valle de Tehuacán-Cuicatlán. Boletín de la Sociedad Botánica de México 67:27-74.

Willing, M. R., M. Kaukman, y R. D. Stevens. 2003. Latitudinal gradients of biodiversity: pattern, process, scale and synthesis. Annual Reviews of Ecology. Evolution Systematics 34:273-309.

Yáñez-Arenas, C., S. Mandujano, y E. Martínez-Meyer. 2014. Predicting the density and abundance of whitetailed deer based on ecological niche theory. Deer Specialist Group IUCN Newsletter 26:20-30.

Summited: March 7, 2015

Review: April 22, 2015

Accepted: May 2, 2015

Associated editor: Consuelo Lorenzo 
FOTO-TRAMPEO DE MAMÍFEROS EN OAXACA

448 THERYA Vol.6(2): 435-448 